\title{
Infrared spectra of ZrC presolar grain analogues
}

\author{
Y. Kimura and C. Kaito \\ Department of Nanophysics in Frontier Project, Ritsumeikan University, Kusatsu-shi, Shiga 525-8577, Japan \\ e-mail: kaito@se.ritsumei.ac.jp \\ Received 4 March 2003 / Accepted 16 June 2003

\begin{abstract}
The origins of spectral feature of stellar source are identified on the basis of laboratory studies. Although $\mathrm{ZrC}$ grains were identified as the crystalline presolar grains in meteorites, the spectral data of $\mathrm{ZrC}$ grains have not been measured in the laboratory. Therefore, $\mathrm{ZrC}$ grains with the same size as the presolar grains were produced in the laboratory. It has been found that the infrared spectrum of $\mathrm{ZrC}$ grains shows broad absorption features at 9.4 and $12.4 \mu \mathrm{m}$.
\end{abstract}

Key words. stars: AGB and post-AGB - stars: carbon - stars: circumstellar matter - ISM: general - infrared: ISM infrared: stars

\section{Introduction}

Presolar grains of carbide have been identified from the anomalous isotopic composition of carbon, nitrogen and silicon in $\mathrm{SiC}$ grains in the Murray carbonaceous meteorite (Bernatowicz et al. 1987). In addition to isotopic measurements, metallic ( $\mathrm{Ti}, \mathrm{Mo}$, and $\mathrm{Zr}$ ) carbides were identified in the 5-200 nm crystalline presolar grains by transmission electron microscopy (TEM) from graphitic spherules in Murchison meteorites (Bernatowicz et al. 1996). These metallic carbides were surrounded by well-graphitized carbon, i.e., many have a composite structure of a core of metallic carbides surrounded by a carbon mantle. Therefore in this paper, composite grains are denoted as core-mantle grains.

The origins of absorption peaks are identified on the basis of laboratory studies. Important factors governing the absorption features are size, shape and structure of the grain (Bohren \& Huffman 1983). Therefore, the production of a presolar grain analogue is important for the identification of observed spectra.

One of the best methods of producing nanometer metallic carbide grains as presolar grains in the laboratory is the smoke experiment, which is a gas evaporation method (Uyeda 1987, 1991). Using the gas evaporation method, solid grains are directly produced from the gas phase. We have produced metallic, oxide and sulfide grains by gas evaporation methods. The growth mechanism of these grains has been elucidated in a series of experiments (Kaito 1978, 1981, 1984, 1985). However, since metallic carbide grains are refractory materials, their production is difficult. TiC core-carbon mantle grain formation was achieved in a previous study by introducing a noble gas

Send offprint requests to: Y. Kimura, e-mail: rp092955@se.ritsumei.ac.jp

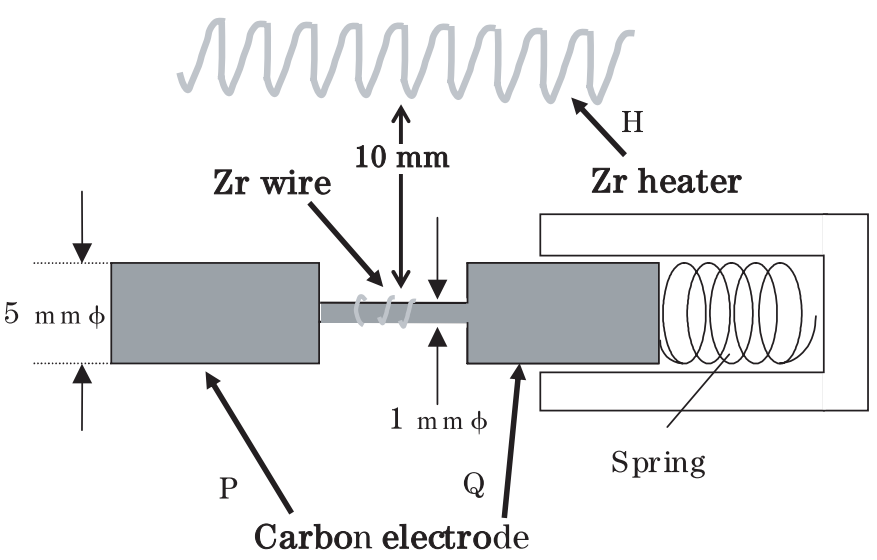

Fig. 1. Experimental method of producing $\mathrm{ZrC}$ core-carbon mantle grains in smoke. Zirconium wire was set around the carbon electrode.

evaporation method (Kimura \& Kaito 2003). In the present experiment, we produced $\mathrm{ZrC}$ core-carbon mantle grains.

Although $\mathrm{ZrC}$ grains were identified in meteorites (Bernatowicz et al. 1996), the spectral data of $\mathrm{ZrC}$ grains have not been measured in the laboratory. Therefore, $\mathrm{ZrC}$ grains were produced in the present experiment. The characteristic spectrum is shown for $\mathrm{ZrC}$.

\section{Experimental methods}

The work chamber used was a glass cylinder $17 \mathrm{~cm}$ in diameter and $30 \mathrm{~cm}$ in height covered with a stainless-steel plate and connected to a high-vacuum exhaust through a valve at its bottom. The present successful method of producing the $\mathrm{ZrC}$ grains is shown schematically in Fig. 1. Two carbon electrodes, $\mathrm{P}$ and $\mathrm{Q}$, with a fine point, were prepared. The end of electrode $\mathrm{Q}$ was pushed with a spring. $\mathrm{Zr}$ wire was set around carbon 


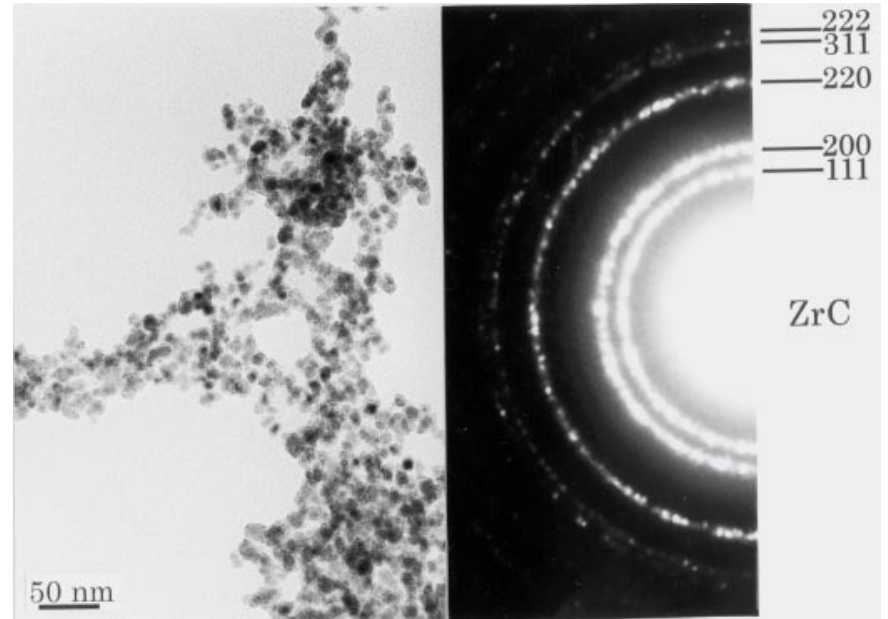

Fig. 2. Typical TEM image and corresponding ED pattern indicate the formation of $\mathrm{ZrC}$ grains.

electrode Q. After the chamber was evaporated to a pressure less than $1 \times 10^{-3} \mathrm{~Pa}$, Ar gas was introduced at $13.3 \mathrm{kPa}$. By heating the carbon electrode in argon gas, the $\mathrm{Zr}$ wire was wet around the fine point of carbon electrode Q. As a result, since evaporation of carbon was suppressed, $\mathrm{ZrC}$ grains were obtained.

When the arc discharge is initiated in inert gas by this method, smoke rising from the evaporation source appears. The evaporated vapor is subsequently cooled and condensed in the gas atmosphere, i.e., solid grains are obtained directly from the gas cloud. Coalescent growth is regarded to be an important process in this method. Coalescence of the smoke particles has been explained in terms of two stages: a surface melting coalescence stage and a liquid-like coalescence stage among solid grains (Kaito 1978). These two stages depend mainly on grain size and temperature. The morphology and size of grains are controlled by the mass density and temperature of the smoke, which themselves depend on the atmospheric gas and gas pressure. By the same method, we succeeded in producing TiC core-carbon mantle grains of the same size as the core of presolar grains (Kimura \& Kaito 2003).

The specimens were examined using a Hitachi H-7100R TEM. High-resolution transmission electron microscopy (HRTEM) images were obtained using a Hitachi H-9000NAR TEM. The infrared spectra of the samples embedded in $\mathrm{KBr}$ pellets were measured with a Fourier-transform infrared spectrometer (Horiba Inc. FT210). The wavelength resolution was $2 \mathrm{~cm}^{-1}$.

\section{Results and discussion}

Figure 2 shows a typical TEM image and corresponding electron diffraction (ED) pattern of the collected specimen obtained by simultaneous evaporation of zirconium and carbon, as shown in Fig. 1. The ED pattern shows the formation of $\mathrm{ZrC}$ grains. A typical HRTEM image of a $\mathrm{ZrC}$ grain is shown in Fig. 3. The lattice spacing and angles between crossed lattices in the image indicate the existence of $\mathrm{ZrC}$ polycrystals, i.e., $\mathrm{ZrC}$ grains were composed of $5 \mathrm{~nm}$ crystallites. The external

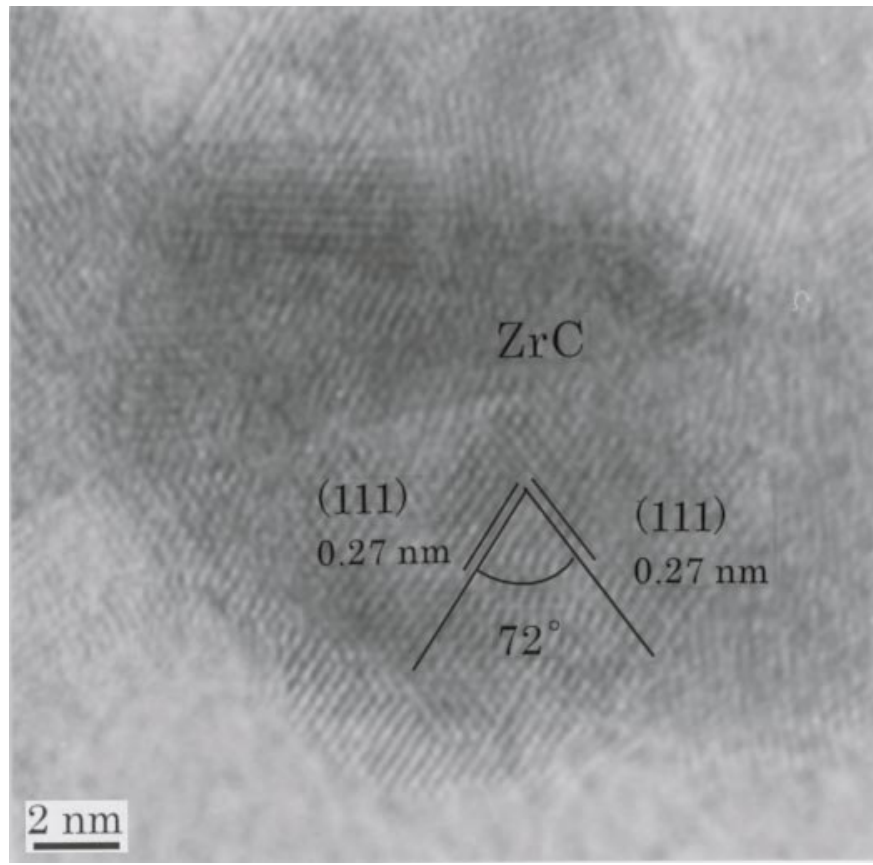

Fig. 3. Typical HRTEM image of a $\mathrm{ZrC}$ grain. The lattice distance and angles between closed lattice images indicate the existence of $\mathrm{ZrC}$ polycrystals.

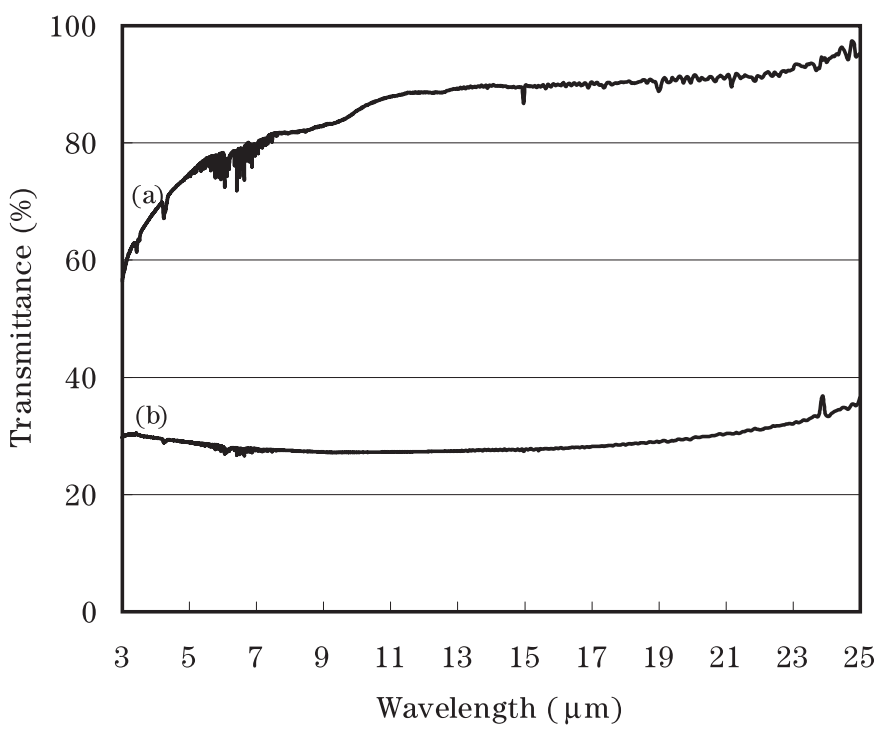

Fig. 4. Infrared spectra of $\mathrm{ZrC}$ grains. a) Spectrum for $\mathrm{ZrC}$ grains shown in Fig. 3. Broad absorption peak at $9.4 \mu \mathrm{m}$ is clearly observed. b) Spectrum of commercial Nilaco ZrC powder shown in Fig. 5.

shape becomes spherical in spite of the NaCl-type structure, because the particles were compounds of crystallites and had many defects. Amorphous carbon grains with a size of $20 \mathrm{~nm}$ were also seen. Therefore, a carbon-rich condition is formed during $\mathrm{ZrC}$ growth. More than $50 \%$ of $\mathrm{ZrC}$ grains were surrounded by a carbon mantle. Zirconium grains are negligible. Figure 4a shows the infrared spectrum of the collected $\mathrm{ZrC}$ grains embedded in $\mathrm{KBr}$ pellets. The spectrum has a broad absorption feature at $9.4 \mu \mathrm{m}$. A very weak hump at the $12.4 \mu \mathrm{m}$ absorption feature is also seen. Amorphous carbon grains were 


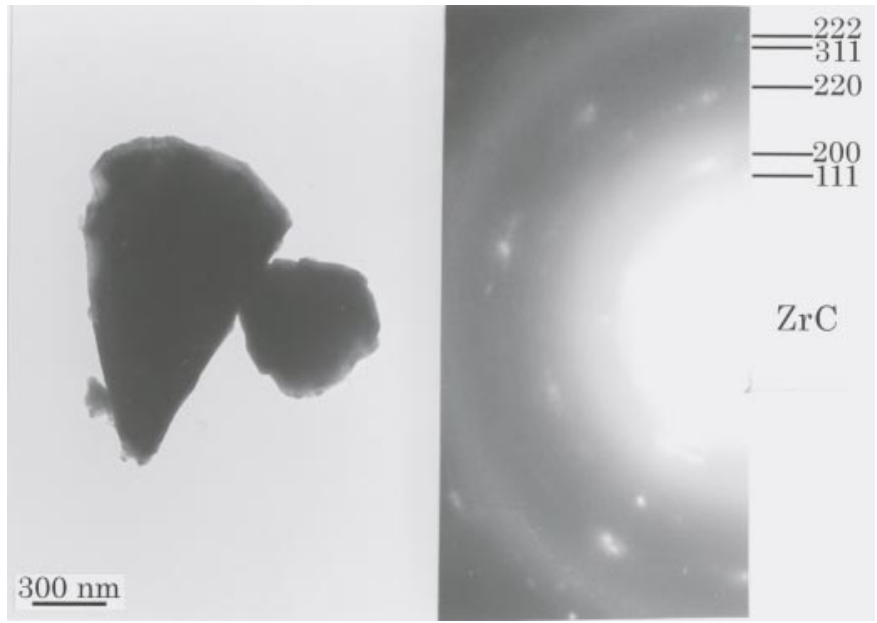

Fig. 5. Typical TEM image and ED pattern of commercial Nilaco $\mathrm{ZrC}$ powder.

produced and their spectra were measured. The only absorption feature was a broad peak at $8.3 \mu \mathrm{m}$; the peaks at 9.4 and $12.4 \mu \mathrm{m}$ did not appear. Therefore, the absorption at 9.4 and $12.4 \mu \mathrm{m}$ was identified to be of $\mathrm{ZrC}$ origin. Since the $\mathrm{ZrC}$ presolar grains found in meteorites are nm-sized and coated with carbon, 9.4 and $12.4 \mu \mathrm{m}$ features correspond to the $\mathrm{ZrC}$ presolar grains.

The infrared spectrum of commercial Nilaco $\mathrm{ZrC}$ powder was never observed within the limits of our measurements (3$25 \mu \mathrm{m}$ ), as shown in Fig. 4b. The size of the measured powder was $0.3-5 \mu \mathrm{m}$ which was separated by dispersion in ethanol. Typical Nilaco ZrC powder grains are shown in the TEM image in Fig. 5. Therefore, the absorption peak may be weakened by the increasing size of $\mathrm{ZrC}$ grains. This indicates that infrared absorption of the NaCl-type structure is weaker than that of any other structure, as discussed with respect to the mixture film of $\mathrm{MgO}$ and $\mathrm{SiO}$ (Suzuki et al. 2000).

The TiC clusters with 27 to 125 atoms show a feature at a wavelength of $20.1 \mu \mathrm{m}$ (von Helden et al. 2000). On the other hand, a recent report on the $\mathrm{TiC}$ spectrum, in which infrared reflectance of TiC bulk material is shown, suggested that $\mathrm{TiC}$ are not responsible for the $20.1 \mu \mathrm{m}$ feature
(Henning \& Mutschke 2001). Although TiC core (50 nm)carbon mantle $(2 \mathrm{~nm})$ grains were thought to be at 9.5 and $12.5 \mu \mathrm{m}, 21 \mu \mathrm{m}$ was not included (Kimura \& Kaito 2003). The $21 \mu \mathrm{m}$ feature of the gas phase TiC cluster by von Helden may be remarkable effect in cluster size (von Helden et al. 2000), as is discussed in a previous paper (Kimura \& Kaito 2003). Therefore, 9.4 and $12.4 \mu \mathrm{m}$ features of $\mathrm{ZrC}$ grains may be due to small grain size. Size dependence of $\mathrm{ZrC}$ grains was very similar to the result of $\mathrm{TiC}$ grains. $\mathrm{ZrC}$ and $\mathrm{TiC}$ have the same crystal structure as $\mathrm{NaCl}$ against covalently bound $\mathrm{SiC}$. Since the absorption of $\mathrm{SiC}$ is strong against as the continuum, the spectra can be easily observed. Consideration of the absorption intensity on bounding and crystal structure may become an important factor. The appearance of the $21 \mu \mathrm{m}$ feature in the $\mathrm{TiC}$ cluster may occur on $\mathrm{ZrC}$ for the very small clusters in gas phases. Since the absorption of $\mathrm{ZrC}$ is very weak compared to the continuum, the feature on $\mathrm{ZrC}$ may be unlikely to be detected by the observational spectrograph techniques in spite of its existence in meteorites.

\section{References}

Bernatowicz, T., Fraundorf, G., Ming, T., et al. 1987, Nature, 330, 728 Bernatowicz, T. J., Cowsik, R., Gibbons, P. C., et al. 1996, ApJ, 472, 760

Bohren, C. F., \& Huffman, D. R. 1983, Absorption and Scattering of Right by Small Particles (Wiley Interscience)

Henning, Th., \& Mutschke, H. 2001, AcSpe A, 57, 815

Kaito, C. 1978, JaJAP, 17, 601

Kaito, C. 1981, JCrGr, 55, 273

Kaito, C. 1984, JaJAP, 23, 525

Kaito, C. 1985, JaJAP, 24, 261

Kimura, Y., \& Kaito, C. 2003, in press

Kubo, R. 1962, J. Phys. Soc. Jpn., 17, 975

Oshima, C., Aizawa, T., Wuttig, M., et al. 1987, Phys. Rev. B, 36, 7510

Suzuki, N., Kimura, S., Nakada, T., et al. 2000, M\&PS, 35, 1269

Uyeda, R. 1987, in Morphology of Crystal, Part B, ed. I. Sunagawa, 369 (Tokyo Terra)

Uyeda, R. 1991, Methods of preparation and technical applications. Prog. in Material Science 35, 1-96 (Pergamon Press)

von Helden, G., Tielens, A. G. G. M., von Heijnsbergen, D., et al. 2000, Science, 288, 313 